Check for updates

The BMJ

Cite this as: BMJ2020;371:m4777 http://dx.doi.org/10.1136/bmi.m4777 Published: 09 December 2020

\section{Covid-19: Oxford vaccine could be $59 \%$ effective against asymptomatic infections, analysis shows}

\author{
Elisabeth Mahase
}

The Oxford and AstraZeneca covid-19 vaccine prevented some asymptomatic covid-19 infections in phase III trials, but the effect was mainly seen in the group who received a half dose first, followed by a full dose, the peer reviewed efficacy results have shown.

The study, published in the Lancet, found that vaccine efficacy against asymptomatic transmission was $59 \%$ in the group that received a half dose followed by a standard dose (seven cases among 1120 participants versus 17 cases among 1127 participants in the control group), but just $4 \%$ in the group that received two standard doses ( 22 among 2168 participants versus 23 among 2223 for the control). The researchers said, however, that as this was a secondary outcome, additional confirmation was still required.

Analysis of the primary outcome-efficacy against symptomatic cases of covid-19-showed that the vaccine was $62.1 \%$ effective in participants who received two standard doses $(n=4440)$ and 90\% effective in those who received a low dose followed by a standard dose $(n=1120)$. This analysis was based on 131 symptomatic cases, of which 30 were in the vaccine arm (27 in the two full doses group, three in the low dose group) and 101 in the control group.

Between April and November 2020, 23848 participants were enrolled to the trial. Half of the participants were given the covid-19 vaccine and the other half given a control (either a meningococcal conjugate vaccine or saline). After a dosing error led to a small group of participants receiving half a dose of the covid-19 vaccine, the researchers-with approval from the Medicines and Healthcare Products Regulatory Agency-added a half dose plus full dose group. ${ }^{2}$ This group, however, did not include adults over 55 years old as the low dose was given in an early stage of the trial, before older adults were recruited.

The interim analysis included 11636 participants (7548 in the UK, 4088 in Brazil, 12\% older adults, and $83 \%$ white). Most were aged $18-55$ years $(82 \%)$; people aged 56 years and older will be studied in future analyses of the trial, the researchers have said.

Merryn Voysey, study author and lead statistician at the Oxford Vaccine Group said, "In future analyses, with more data included as it becomes available, we will investigate differences in key subgroups such as older adults, various ethnicities, doses, and timing of booster vaccines, and we will determine which immune responses equate to protection from infection or disease."

Alongside asymptomatic infections, another secondary outcome of the trial was prevention of severe disease. The paper found that, from 21 days after the first dose, 10 cases of patients being admitted to hospital for covid-19 were reported, all of which were in the control arm. Two of these cases were classified as severe, including one death.

The trial also monitored safety for a median of 3.4 months in all 23745 participants from the UK, Brazil, and South Africa. Of these participants, 168 experienced a total of 175 severe adverse events over the period, but 172 of these were deemed unrelated to the covid-19 or control vaccines. Of the three events remaining, one was in the control group (a case of haemolytic anaemia), one was in the covid-19 vaccine group (a case of transverse myelitis considered possibly related to the vaccine), and the third was a case of severe fever $\left(>40^{\circ} \mathrm{C}\right)$ reported in a participant who remains masked to group allocation and recovered rapidly without an alternative diagnosis or being admitted to hospital. All three participants have recovered or are recovering and remain part of the trial.

\section{Sinopharm vaccine results}

Meanwhile, another covid-19 vaccine candidate-developed by state owned Chinese company Sinopharm and the Beijing Institute of Biological Products-has reportedly been found to be $86 \%$ effective against covid-19. ${ }^{3}$ The United Arab Emirate's Ministry of Health and Prevention released a statement on 9 December claiming that it had reviewed the interim analysis of a phase III trial that is ongoing in the country and has enrolled 31000 people. They reported that the inactivated vaccine cases of the disease and had no serious safety concerns. But no further details have been released. In September, this vaccine was granted emergency use authorisation by the United Arab Emirates and was rolled out to frontline workers.

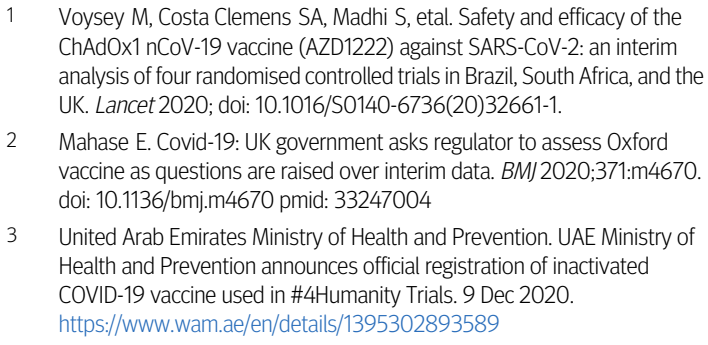

Voysey M, Costa Clemens SA, Madhi S, etal. Safety and efficacy of the ChAdOx1 nCoV-19 vaccine (AZD1222) against SARS-CoV-2: an interim analysis of four randomised controlled trials in Brazil, South Africa, and the UK. Lancet 2020; doi: 10.1016/S0140-6736(20)32661-1.

2 Mahase E. Covid-19: UK government asks regulator to assess Oxford vaccine as questions are raised over interim data. BMJ2020;371:m4670. doi: 10.1136/bmj.m4670 pmid: 33247004

3 United Arab Emirates Ministry of Health and Prevention. UAE Ministry of Health and Prevention announces official registration of inactivated COVID-19 vaccine used in \#4Humanity Trials. 9 Dec 2020. https:/www.wam.ae/en/details/1395302893589 This article is made freely available for use in accordance with BMJ's website
terms and conditions for the duration of the covid-19 pandemic or until otherwise
determined by BMJ. You may use, download and print the article for any lawful,
non-commercial purpose (including text and data mining) provided that all copyright notices and trade marks are retained. was $100 \%$ effective at preventing moderate and severe 